\title{
Power and Signal Integrity and Electromagnetic Emission; the balancing act of decoupling, planes and tracks
}

\author{
Thales Netherlands \\ Hengelo, The Netherlands \\ frank.leferink@nl.thalesgroup.com
}

Frank B.J. Leferink

\author{
University of Twente \\ Enschede, The Netherlands \\ frank.leferink@utwente.nl
}

$$
Z_{\text {noise }}=Z_{g}=R_{g}+\mathrm{j} \omega L_{g_{\text {net }}}
$$

printed circuit board is often the cause of unwanted radiated emission. Power supply planes attribute to the noise voltage. By replacing the power supply planes by tracks, the noise voltage in the reference or ground can be considerably reduced, which leads to a dramatic reduction of the radiated electromagnetic fields. This has been shown using transmission line equations for a general two-conductor line. Simulation and measurements results confirm the beneficial effect of removing power planes. A reduction of $50 \mathrm{~dB}$ in radiated electromagnetic field strength was obtained. The question was raised whether (or not) the signal integrity is negatively influenced by using power tracks instead of planes. Several printed circuit boards have been built and measured, showing that signal integrity remains while reduction of radiated emission is achieved.

\section{INTRODUCTION}

Unwanted radiated electromagnetic fields are often created by unwanted antenna currents, generated by a noise voltage in the reference (ground) conductor. The focus has been on reducing the net partial inductance of the reference so that the noise voltage is reduced (for example [1],[2],[3],[4],[5] and many, many others). This conventional model is not valid for high frequencies where the longitudinal dimensions of a product are in the order of magnitude of a wavelength. The conclusion drawn is that a (unbalanced) transmission line should be, geometrically seen, asymmetrical [6], [7]. This assumption can be extended to power planes too, leading to the conclusion than power planes should be omitted in order to reduce radiated emission. This effect has been presented in [8]. The cause of unwanted longitudinal noise voltage is described shortly, and applied to power planes. New printed circuit boards have been built to measure the effects of power planes, power tracks, various decoupling schemes on radiated emission as well as signal integrity.

\section{NOISE VOLTAGE}

The conventional model for the open circuit longitudinal noise voltage $U_{\text {noise }}$ due the signal current flow through a finite impedance of the reference (ground) is

$$
U_{\text {noise }}=Z_{\text {noise }} I_{\text {sig }}
$$

with with $R_{g}$ as the resistance of the reference (ground) and $L_{g_{\text {net }}}$ as the net partical inductance of the reference.

The conventional model (1) would imply a higher noise voltage with increasing frequency. This does not correspond with actual situations where at higher frequencies the noise voltage is flat with a $\sin x / x$ behavior.

In [6] and [7] a better model has been developed using transmission line theory, called Signal to Noise Transformation (SNT), resulting in:

$$
U_{\text {noise }}=\frac{Z_{g} U_{s}}{Z_{s}+Z_{c}} \frac{1}{\gamma_{s}} \frac{\left[\left(e^{-\gamma_{s} p}-1\right)+\Gamma_{l} e^{-2 \gamma_{s} p}\left(e^{-\gamma_{s} q}-1\right)\right]}{1-\Gamma_{l} \Gamma_{s} e^{-2 \gamma_{s} p}}
$$

with $U_{s} \quad$ signal voltage

$Z_{s} \quad$ source impedance

$p \quad$ length transmission line

$q \quad$ length common in reference

and

$$
\Gamma_{s}=\frac{Z_{s}-Z_{c}}{Z_{s}+Z_{c}} \quad \Gamma_{l}=\frac{Z_{l}-Z_{c}}{Z_{l}+Z_{c}}
$$

If the length of the transmission line $p$ and the part shared with the environment $q$ is less than a fraction of a wavelength

$$
\begin{gathered}
p, q<\lambda / 2 \pi \sqrt{\varepsilon_{r}} \quad \text { and } \quad \text { if } \quad R_{g} \leq \omega L_{g_{\text {net }}}, \text { and if } \\
Z_{s}+Z_{l} \gg \omega L_{g_{\text {net }}} \text { then (3) can be simplified to } \\
\frac{U_{\text {noise }}}{U_{s}} \approx \frac{\mathrm{j} \omega L_{g_{\text {net }}}}{Z_{s}+Z_{l}}
\end{gathered}
$$

which is similar to the conventional noise voltage equations. The interesting part is however at high frequencies, when the length of the transmission line $p$ and the part shared with the environment $q$ is in the order of or longer than a wavelength, i.e. $p, q \geq \lambda / 2 \pi \sqrt{\varepsilon_{r}}$. Then we need the complete SNT equation (3). Due to the forward and backward travelling waves the noise voltage is nearly flat with increasing frequency with addition and subtraction effects (resonances and anti-resonances). $\gamma_{s} \quad$ propagation constant 
If $Z_{l}=Z_{s}=Z_{c}$ then (3) reduces to

$$
\frac{U_{\text {noise }}}{U_{s}}=\frac{Z_{g}}{2 Z_{c}} \frac{1}{\gamma_{s}}\left[\mathrm{e}^{-\gamma_{s} q}-1\right]
$$

and the amplitude is maximal if $\gamma_{s} q=(2 n-1) \pi$ for $n=0,1,2 \ldots$, i.e. if $q=(n-1 / 2) v / f$, resulting in the maximal noise voltage of

$$
\frac{U_{\text {noise }}}{U_{s}}=\frac{L_{g_{\text {net }}}}{L_{\text {loop }}}
$$

In other words, the longitudinal noise voltage developed in the ground conductor is determined by the net partial inductance of that ground conductor divided by the total loop inductance of the transmission line. This means that a (unbalanced) transmission line should be, geometrically seen, asymmetrical to reduce the noise voltage [6], [7].

\section{CONVENTIONAL POWER DISTRIBUTION}

Power distribution networks are generally low impedance conductors (planes) so that the longitudinal voltage drop between two units is minimal. A cross-section of a typical 4layer multi-layer PCB is drawn in Figure 1, left. VCC denotes the power plane, Sig1 and Sig2 the signal track planes and GND the ground plane. The instantaneous signal current generated by the device in the signal line has to be supplied by the decoupling capacitor. This current will generate a noise voltage in the ground system through the net partial inductance of the ground plane. In case of a lack of a nearby placed decoupling capacitor the instantaneous signal will be supplied by the power planes.
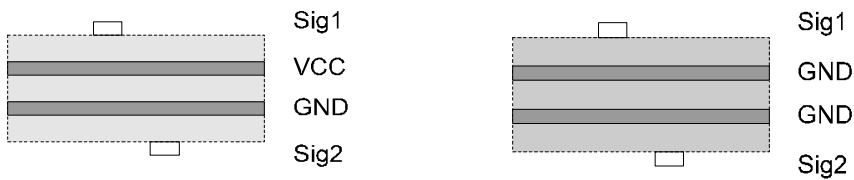

Figure 1: Cross-section of $\mathrm{PC}$ with symmetrical and asymmetrical power distribution system

The total noise voltage is the sum of $U_{\text {noise, power }}$,

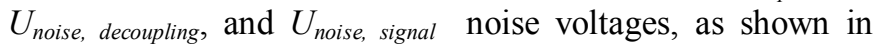
Figure 4. The signal noise voltage $U_{\text {noise, signal }}$ can be reduced by using large ground planes and nearby placed small signal tracks, in order to increase the asymmetry [8], [10], [11]. The noise voltage due to the decoupling current can be reduced by placing the decoupling capacitor nearby, without making use of the reference (ground) plane.

The noise voltage $U_{\text {noise, power }}$ is, as a first order assumption, created by the instantaneous supply current $I_{i s}$ and the impedance of the reference (ground) plane $Z_{\text {noise }}$ :

$$
U_{\text {noise, power }}=Z_{\text {noise }} I_{\text {is }}
$$

This simple, conventional, model leads to the same limited conclusions as drawn in the last two decade for the noise voltage due to signal line currents: reduce the impedance of the reference to reduce the noise voltage. We have shown that the noise voltage at high frequencies is not determined by the absolute impedance of the reference plane, but by the geometrical asymmetry of the transmission line system. This observation would imply the use of power supply tracks instead of supply planes. Eventually small ferrite beads can be placed in series with the power track so that the inductance will increase even more. This has been drawn in Figure 1, right.

\section{SimULATION RESULTS}

A simulation has been carried out. The transmission line was assumed to be $300 \mathrm{~mm}$ long, $50 \mathrm{~mm}$ wide ground plane. If a $50 \mathrm{~mm}$ wide power supply plane is used then the characteristic impedance $Z_{c}$ is approximately $8 \Omega$. Two simulations have been carried out for this stack-up. The first one was with a resistive source and load impedance, $Z_{s}=5 \Omega$ and $Z_{l}=100 \Omega$ respectively. The second was with additional capacitive source and load impedances of $10 \mathrm{nF}$. The noise voltage developed in the ground plane is shown in Figure 2, using (3). The simulation with a circuit analysis tool gave the same result.

A simulation using a $3 \mathrm{~mm}$ wide supply track has been carried out too. Then the characteristic impedance is $50 \Omega$. The noise voltage is also drawn in Figure 2.

A capacitive loads results in a highly resonant behavior. Resistive source and load impedances damp the resonances. But if a power supply track instead of a power supply plane is used the noise voltage amplitude is decreased over the whole frequency range.

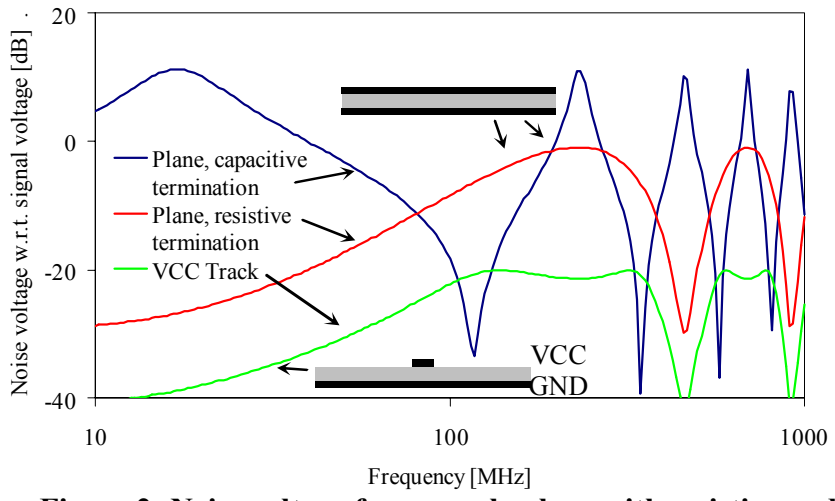

Figure 2: Noise voltage for a supply plane with resistive and capacitive termination, and the supply track situation.

\section{EXPERIMENTS WITH PCB TRANSMISSION LINES}

Measurements have been carried out to validate the power isolation concept. A $20 \mathrm{MHz}$ clock generator was placed on a $50 \mathrm{~mm}$ wide ground plane. The output was loaded with a $470 \Omega$ resistor parallel with a $22 \mathrm{nF}$ capacitor and both connected to the ground plane. The clock generator was supplied via a $50 \mathrm{~mm}$ wide VCC supply plane. The supply was taken from a $9 \mathrm{~V}$ battery which was stabilised by a 78L05 voltage stabiliser. The radiated electric field strength has been measured at $20 \mathrm{MHz}, 40 \mathrm{MHz}, 60 \mathrm{MHz}$ etc.

Several variants have been implemented:

1. Supply via a plane, no decoupling.

2. Supply via a track, no decoupling 
3. Track, decoupling capacitor near clock.

4. Track, series inductance and decoupling capacitor.

Results are shown in Figure 3.

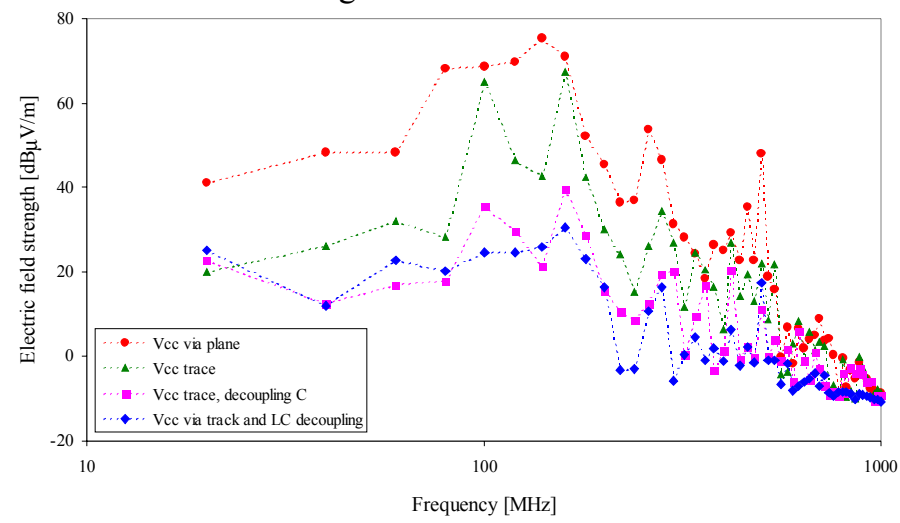

Figure 3: Electric field strength of a basic test $\mathrm{PCB}$ with power planes and tracks

The effect of preventing the power supply current to flow through the ground plane is obvious when comparing the measured data; A reduction of the field strength of approximately $50 \mathrm{~dB}$ can be observed. The influence of resonances, peaks around 100 and $200 \mathrm{MHz}$, can be seen.

\section{EXPERIMENTS WITH PCB WOTH CLOCK AND DRIVER IC}

A power supply (LM340-5 and capacitors), a clock generator, an octal driver (74ACT541) and an actual load (8 SMD resistors) are placed on a printed circuit board (PCB), as shown in Figure 4 and 5 . The power supply was via a ground plane (Figure 4) and a power track (Figure 5). For reference, a PCB with signal traces above a ground plane has been measured too. The radiated emission level has been measured with an absorbing clamp. To reduce external influences the PCBs were placed in metallic box.

Measurements have been performed at every $10 \mathrm{MHz}$ harmonic between $10 \mathrm{MHz}$ and $3000 \mathrm{MHz}$. The measured power is shown in Figure 7. It shows

- PCB with signal track above a ground plane, so that the ground plane conducts the momentary return current, which causes a noise voltage, and the noise volatge causing an antenna current in the attached wire.

- PCB with power plane, wich conducts only a limited amount of the momentary current current due to the local decoupling capacitor+resistor.

- PCB with power track, wich again conducts only a limited amount of the momentary current current due to the local decoupling capacitor+resistor.

The curves show the measured power, with the absorbing clamp, at $10 \mathrm{MHz}$ intervals (thin line). A 2-point moving average curve (thick line) has been added for readability. Note that in this measurement the devices have been decoupled properly, so that only a limited amount of momentary (highfrequency) current is flowing through the power plane/track. The beneficial effect of power tracks instead of planes, as shown in Figure 7, is obvious.

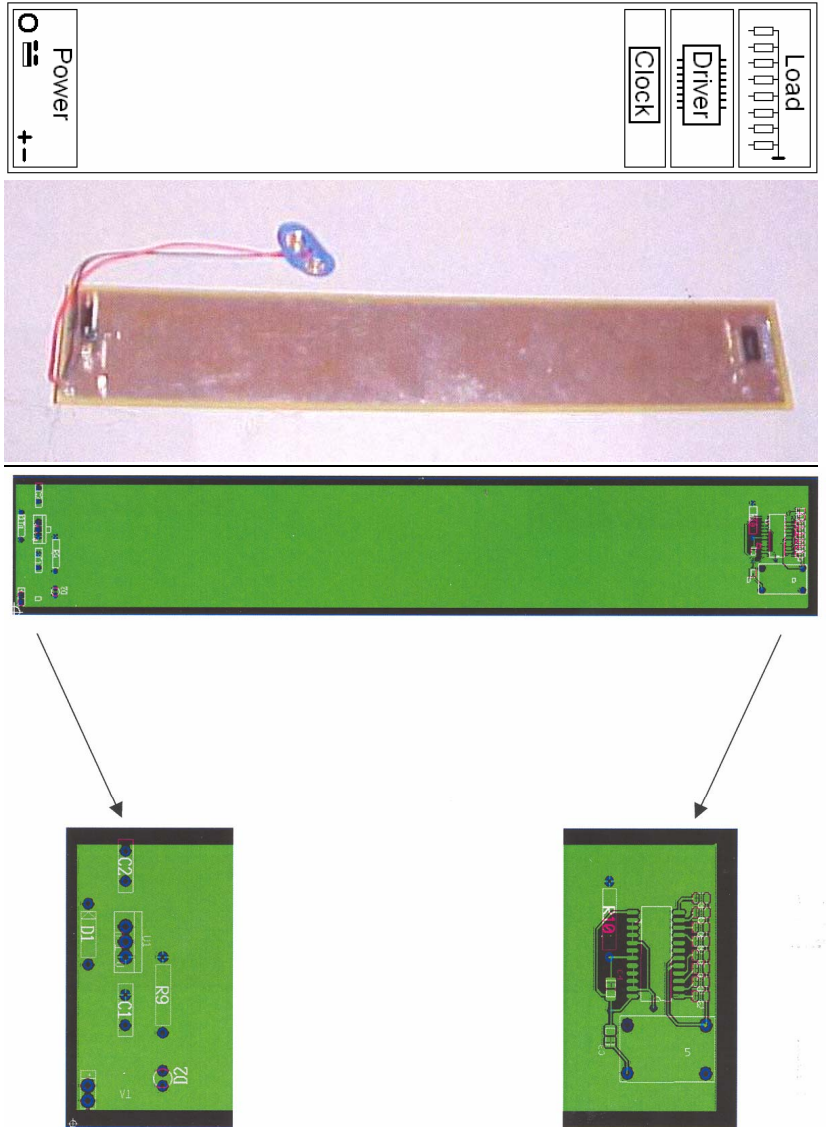

Figure 4: Printed circuit board 1, power plane
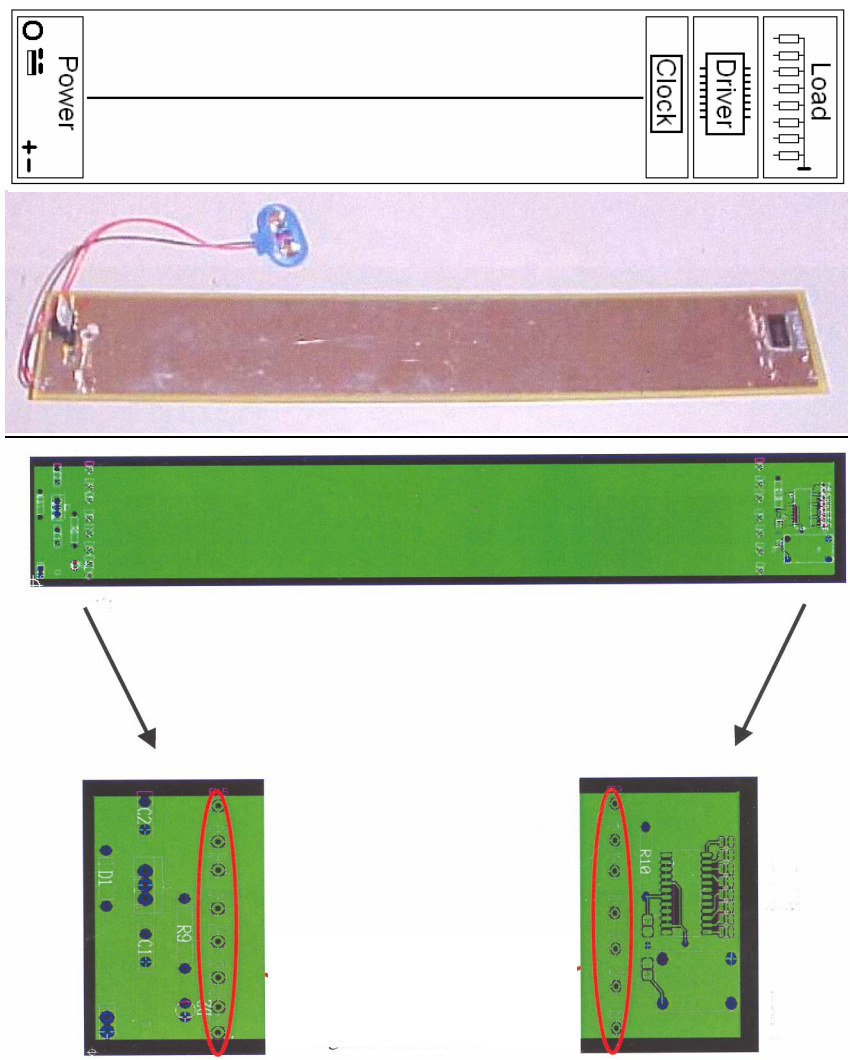

Figure 5: Printed circuit board 2, power track 


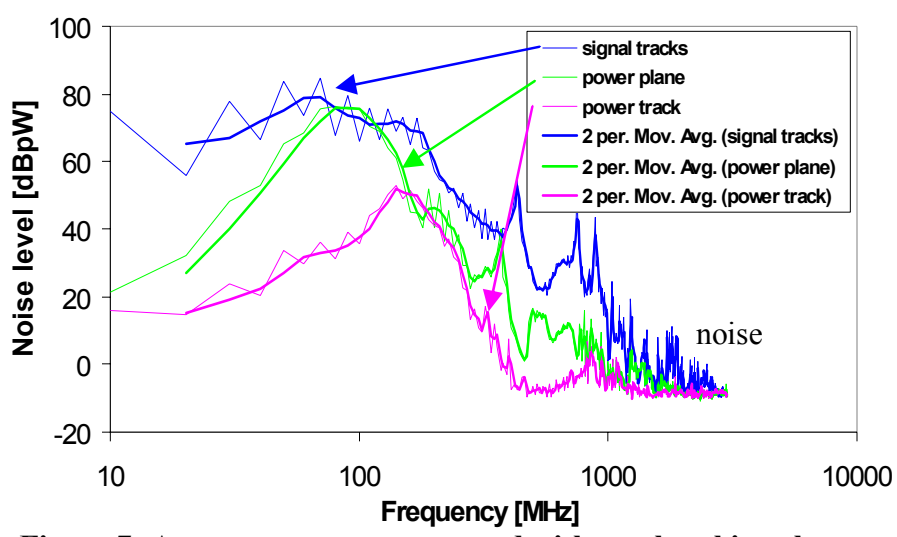

Figure 7: Antenna currents measured with an absorbing clamp of a PCB with signal tracks above a ground plane, a PCB with power plane and a PCB with power track

\section{POWER AND SIGNAL INTEGRITY}

Power planes are an established issue and many people believe in the positive effects of power planes. By removing power planes, you are removing confidence in the EMC performance, and especially the power and signal integrity performance. Many people believe that the power plane - ground plane combination is an excellent high frequency capacitor which is very efficient for decoupling purposes. A capacitor is however nothing more than a storage facility of charge, i.e electrons. If energy is needed, these electrons have to travel. The speed of electrons in a PCB is approximately $1.510^{8} \mathrm{~m} / \mathrm{s}$. If a circuit needs energy during its rise-time, in say $100 \mathrm{ps,}$ then electrons within a distance of $15 \mathrm{~mm}$ are capable to deliver the energy! A large power plane has thus limited effect in delivering high speed energy.

Seeing is believing. The quality of the output signal of the driver IC was measured, for the power plane and the power track delivery network. The results are very promising: the large scale effects shown in Figure 8 show a very similar result for both track and plane, and even a suggestion that the signal track situation delivers faster the signal to the load. This is confirmed in the detailed Figure 9. The power track gives a very nice output signal of the driver IC, towards the load.

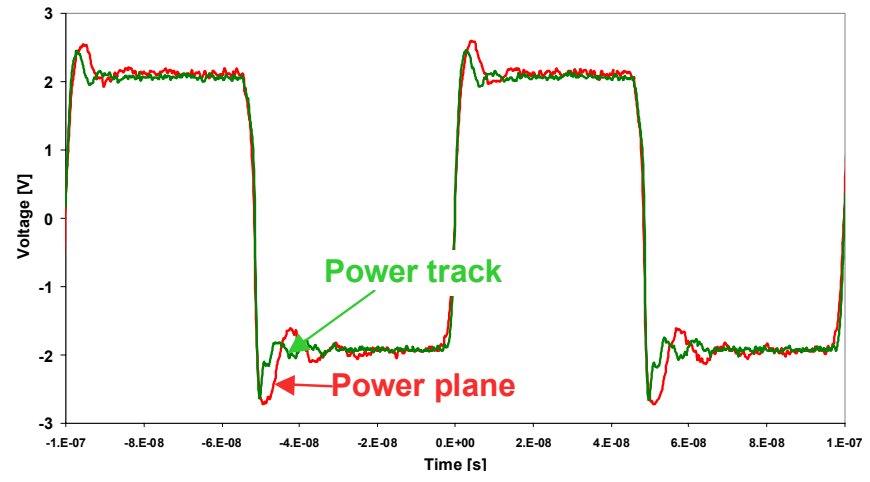

Figure 8: Signal integrity

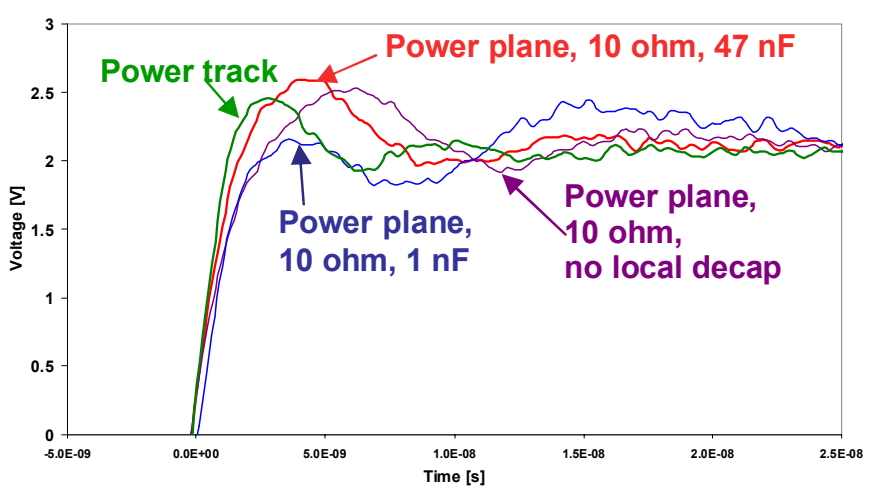

Figure 9: Signal integrity

The impact on the quality of the power supply has been measured too, shown in Figure 10. The power plane without additional decoupling or local stabilization capacitor is worse. The power plane with a $10 \Omega$ resistor and $47 \mathrm{nF}$ capacitor is best, the signal track is second.

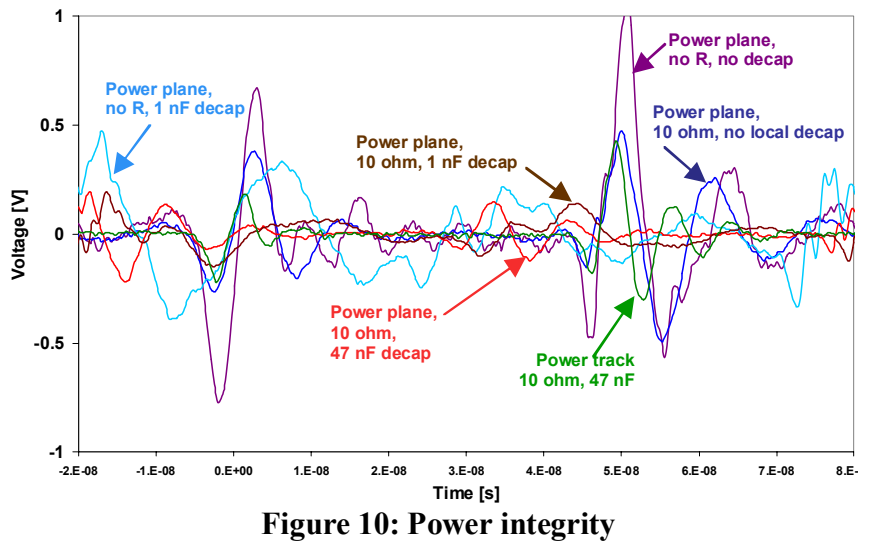

DECOUPLING

It is already mentioned that the power-ground plane structure is not adequate in terms of speed to deliver the energy needed by modern electronic (digital) circuitry. It is however also not adequate in terms of amount of energy. Assume the area below a device with some extra space, say $30 \times 30 \mathrm{~mm}$. The distance between the planes could be something like $100 \mu \mathrm{m}$. The capacitive effect for this area is then something like 200 $\mathrm{pF}$ for FR-4 permittivity. A driver IC can drive loads of 10 $\mathrm{nF}$, so it is obvious that the energy in the power-ground plane structure is inadequate. We need local decoupling capacitors. A better term would be local stabilisation, because the capacitor stabilises the power supply on a fixed level. Decoupling is more on preventing the power current to flow through the whole system. We should focus on a minimum stabilisation for power quality and a maximum decoupling for reduced radiated emission. The impact of decoupling and the use of power track and planes on the unwanted radiated emission have been measured. Results are shown in Figure 11.

The power track PCB shows the lowest radiated emission. The power plane PCB all result in higher emission levels. The plane PCB with a local stabilizing capacitor of $47 \mathrm{nF}$ and a series decoupling resistor of $10 \Omega$ gives the lowest emission 
level. The worse the stabilizer and decoupling, the higher the emission level. This effect is expected, because the higher the high-frequency power current, the higher the noise voltage, and the higher the emission level, see equation (3).

Note the difference in measured values: over $60 \mathrm{~dB}$.

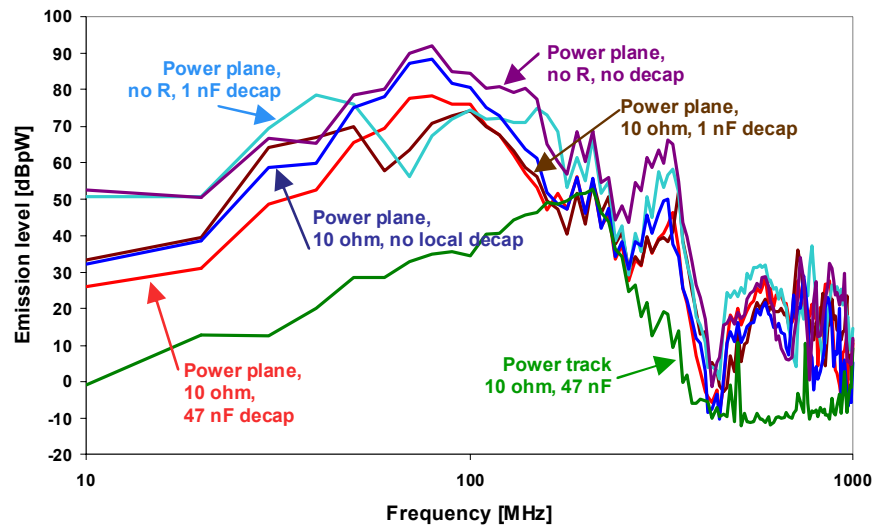

Figure 11: Effect of decoupling on EMI

\section{CONCLUSION}

The noise voltage, the main cause of unwanted radiated electromagnetic fields, can be reduced by creating geometrically asymmetric transmission lines. This asymmetry should also be used for power supply systems. This will imply the removal of power planes. The beneficial effect on the noise voltage due to the skipped power supply plane has been analysed. Simulation results confirm the advantageous effect. Several radiated electric field measurements have been performed, showing a huge reduction of the radiated emission levels. The effect on the integrity of the functional signals and the power integrity has been investigated experimentally. The signals and power supply are not distorted when using tracks instead of planes. The effect of various decoupling topologies on the radiated emission has been measured showing again that the supply track topology is better compared to the supply plane. It has been shown that the power and signal integrity can be maintained when using power tracks instead of planes and still reduce unwanted electromagnetic emission considerably.

\section{REFERENCES}

[1] H.W. Ott, "Controlling EMI by Proper Printed Wiring Board Layout", EMC Zurich 1985, 6th Int. Zurich Symp. and Techn. Exh. on EMC, Zurich, Switzerland, pp. 127-132, 1985

[2] C.R. Paul, "Modeling Electromagnetic Properties of Printed Circuit Boards", IBM Journal Research and Development, vol, vol. 33, nr. 1, pp. 33-49, 1989

[3] F.B.J Leferink, M.J.C.M. van Doorn, "Inductance of Printed Circuit Board Ground Planes", IEEE Int. Symp. on EMC, Dalles, TX, pp. 327-329, 1993

[4] J.L. Drewniak, T.H. Hubing, T.P. VanDoren, "Investigation of Fundamental Mechanisms of Common-Mode Radiation from Printed Circuit Boards with Attached Cables", IEEE Int. Symp. on EMC, Chicago, IL, pp. 110-115, 1994

[5] D.M. Hockanson, J.L. Drewniak, T.H. Hubing, T.P. VanDoren, S. Fei, W.L. Cheung, "Quantifying EMI Resulting From Finite-
Impedance Reference Planes", IEEE Trans. on EMC, vol. 39 , nr. 4, Nov. 1997, pp. 286-97, 1997

[6] F.B.J. Leferink, "Preventing Electromagnetic Interference From Integrated Circuits and Printed Circuit Boards Using Computer Simulation", Report EL BSC 92N138, Twente University of Technology, 1992

[7] F.B.J. Leferink, "Reduction of Radiated Electromagnetic Fields by Creation of Geometrical Asymmetry, PhD. Thesis University of Twente, 2001, ISBN 90-365-1689-7

[8] F.B.J. Leferink, W.C. van Etten, Reduction of Radiated Electromagnetic Fields by Removing Power Planes, IEEE Int. Symp. On EMC, Santa Clara, CA, 2004

[9] F.B.J. Leferink, "Inductance Calculations, Methods and Equations", IEEE Int. Symp. on EMC, Atlanta, GA, pp. 16-22, 1995

[10] F.B.J. Leferink, "Inductance Calculations, Experimental Investigations", IEEE Int. Symp. on EMC, Santa Clara, CA, pp. 235240, 1996

[11] F.B.J. Leferink, "Reduction of Printed Circuit Board Radiated Emission”, IEEE Int. Symp. on EMC, Austin, TX, pp. 431-438, 1997

[12] B. Danker, "The Decoupling of DC Supply Lines", EMC'94 Roma, pp. 177-183

[13] M. Coenen, "Optimising IC Decoupling, for Performance and EMI Levels", Electronic Product Design, vol. 17, nr. 1, Jan 1995, pp. 26-34, 1995

[14] J. Fan, Y. Ren, J. Chen, D.M. Hockanson, H. Shi, J.L. Drewniak, T.H. Hubing, Th. P. van Doren, R.E. DuBroff, "RF Isolation Using Power Islands in DC Power Bus Design “, IEEE Int. Symp. on EMC, Seattle, WA, pp. 838843, 1999

[15] Y. Fukumoto, S. Nakamura, O. Wada, R. Koga, "A Design Method of Decoupling Circuits for a Digital PCB to Reduce High Frequency Current on Power and Ground Planes", EMC'99 Tokyo, Int. Symp. on EMC, Tokyo, Japan, pp. 9-12, 1999

[16] J. Held, Th. Wolf, "Optimized Decoupling Concepts for Digital VLSI Circuits", IEEE Int. Symp. on EMC, Montreal, Canada, pp. 904-909, 2001

[17] T.H. Hubing, J.L. Drewniak, T.P. VanDoren, D.M. Hockanson, "Power Bus Decoupling on Multilayer Printed Circuit Boards", IEEE Trans. on EMC, vol. 37, nr. 2, May 1995, pp. 155-66, 1995

[18] T. Hubing, J. Chen, J. Drewniak, T. van Doren, Y. Ren, J. Fan, R. DuBroff, "Power Bus Noise Reduction Using Power Islands in Printed Circuit Board Designs", EMC'99 Tokyo, Int. Symp. on EMC, Tokyo, Japan, pp. 1-8, 1999

[19] L.P. Janssen, "Reducing the Emission of Multi-Layer PCBs by Removing the Supply Plane", EMC Zurich 1999, 13th Int. Zurich Symp. and Techn. Exh. on EMC, Zurich, Switzerland, pp. 639-644

[20] S. Radu, D. Hockansen, "An Investigation of PCB Radiated Emissions from Simultaneous Switching Noise", IEEE Int. Symp. on EMC, Seattle, WA, pp. 893-898, 1999

[21] H. Sasaki, T. Harada, "A New Decoupling Technique for Suppressing Radiated Emissions Arising from Power Bus Resonance of Multilayer PCBs", EMC'99 Tokyo, Int. Symp. on EMC, Tokyo, Japan, pp. 17-20, 1999

[22] H. Sasaki, T. Harada, T. Kuriyama, "A New Decoupling Circuit for Suppressing Radiated Emissions due to Power Plane Resonance', IEICE Trans. Comm., vol. E85-B, no. 5, May 2002, pp. 1031-1037 